\title{
HEMORRAGIA INTRACEREBRAL ESPONTÂNEA
}

\section{ESTUDO RETROSPECTIVO DE 72 CASOS OPERADOS}

\author{
JOSÉ CORREIA DE FARIAS BRITO*, VALDIR DELMIRO NEVES**, RONALD DE LUCENA FARIAS**, \\ CESAR RIBEIRO FERREIRA***, JOSÉ ALBERTO GONÇALVES DA SILVA**
}

\begin{abstract}
RESUMO - Realizamos estudo retrospectivo de 72 casos de hemorragia intracerebral espontânea (HICE) operados no período de 1970 a 1999. Foram excluídos do grupo os casos de hemorragias cerebrais decorrentes de traumatismos cranianos, tumores, malformações vasculares e doenças hematológicas. A idade variou de 20 a 81 anos com média de 58,2 anos. A maioria dos pacientes era do gênero masculino e de cor branca. Em um terço deles, a pressão arterial estava elevada, e os hematomas lobares ocorreram em maior número de vezes que os ganglionares. A sintomatologia dominante resultou do aumento da pressão intracraniana e das lesões cerebrais focais. Em 52 (72,7\%) casos, foi possível realizar tomografia computadorizada de crânio. A avaliação dos resultados cirúrgicos foi dirigida para cada década, em separado, mostrando taxas de mortalidade com significativas diferenças.
\end{abstract}

PALAVRAS-CHAVE: hemorragia intracerebral espontânea, hematoma intracerebral, tomografia computadorizada, hipertensão arterial, coma, hipertensão intracraniana.

\section{Primary intracerebral hemorrhage: retrospective study of 72 operated cases}

ABSTRACT - We analysed 72 cases of primary intracranial hemorrhage surgically treated from 1970 to 1999. The hemorrhages were diagnosed by computerized axial tomography in 52 pacients. Most hematomas were situated in the cerebral hemispheres (30 percent in thalamus-basal ganglia region and 50 percent in the subcortical matter). There were 10 patients with cerebellar hemorrhage. Hypertension (based in blood pressure recordings in the hospital and history) was found in 24 patients ( 33 percent). The most frequent findings were coma, intracranial hypertension and hemimotor deficit. The death rate registred was 27.7 percent; however, it was found a significant difference in the mortality index when considering the 70-79 decade (62.7 percent) and the 90-99 decade (20.7 percent). A critical analysis was made about depth hematomas, consciousness state and intracranial hypertension with herniation related to surgical procedure.

KEY WORDS: primary intracerebral hemorrhage, CT scan, arterial hypertension, coma, intracranial hypertension.

O termo hemorragia intracerebral primária ou espontânea (HICE) é comumente empregado para designar sangramentos que ocorrem na ausência de traumatismos cranianos, malformações arteriovenosas, tumores, doenças hematológicas e infartos isquêmicos ${ }^{1-5}$. Todavia, não existe opinião unânime para esta conceituação e, por isso, alguns autores, baseados na frequente associação da hemorragia à hipertensão arterial ou devido à maior ocorrência na região dos gânglios da base, preferirem usar expressões como hemorragia hipertensiva ou putaminal ${ }^{6-8}$.

A HICE representa cerca de $10 \%$ das doenças cerebrovasculares. É mais frequente na faixa etária de 50 a 70 anos, predomina no gênero masculino e observa-se maior susceptibilidade na raça

Serviço de Neurologia e Neurocirurgia do Hospital Santa Isabel: *Neurologista, **Neurocirurgião, ***Radiologista. Aceite: 23-fevereiro-2000.

Dr. José Alberto Gonçalves da Silva - Av. Minas Gerais 1150 - 58030-092 João Pessoa PB - Brasil 
negra ${ }^{3,69-11}$. Bruno et al. ${ }^{12}$ realizaram estudo comparativo entre 39 pacientes hispânicos e 47 não hispânicos, todos de cor branca e encontraram hemorragias em número duas vezes maior no grupo de origem espanhola.

Nosso propósito, na apresentação deste estudo, é analisar um grupo de pacientes operados de HICE, levando em consideração a época do atendimento, a qual, achamos, influenciou na decisão e nos resultados do tratamento.

\section{MÉTODO}

Realizamos estudo retrospectivo de 72 pacientes operados de HICE, no Serviço de Neurologia e Neurocirurgia do Hospital Santa Isabel, no período de 1970 a 1999. O paciente mais jovem do grupo tinha 20 anos de idade e o mais velho 81 , sendo a faixa etária média de 58,2 anos. Observou-se amplo predomínio do gênero masculino, não havendo registro de idade nos prontuários de dois pacientes (Tabela 1). Mais da metade dos pacientes era de cor branca e apenas dois apresentavam características do grupo negróide. Não foram incluídos no grupo os casos de hemorragias causadas por traumatismos cranianos, malformações vasculares, tumores e doenças hematológicas. Todos os pacientes foram examinados durante a admissão e apresentavam características clínicas sugestivas de doença cerebrovascular aguda.

Os dados clínicos foram registrados segundo a presença de hipertensão intracraniana ou de sinais focais decorrentes da compressão local exercida pelo hematoma. Em 37 (51,3\%) casos, a suspeita diagnóstica foi feita com ajuda de angiografia cerebral que mostrou, em 25 (67,5\%) deles, desvios arteriais e áreas avasculares típicos de lesões com efeito de massa. Com a introdução da tomografia computadorizada de crânio (TCC) em nosso meio, o diagnóstico, a localização e o volume da hemorragia puderam ser obtidos em 52 (72,7\%) casos.

A técnica cirúrgica consistiu no emprego de craniotomia osteoplástica em 35 casos e de craniectomia em 37 outros. O emprego de microscópio foi introduzido nos pacientes operados no período 1990-1999. A derivação ventriculoperitoneal foi necessária em um paciente.

A avaliação do grupo sobrevivente foi baseada no grau da capacidade funcional readquirida. Os fatores de risco como hematomas profundos, coma, distúrbios respiratórios e as idades entre a quinta e sexta décadas, estavam presentes, isoladamente ou associados, nos 20 casos de óbito (Tabela 2).

\section{RESULTADOS}

Em $24(33,3 \%)$ pacientes, a pressão arterial apresentava cifras elevadas. A localização dos hematomas (Tabela 3), mostrada pela TCC, predominou nos hemisférios cerebrais, sendo $17(31,4 \%)$ situados na região dos gânglios da base e 27 (50,0\%) em áreas lobares. Um paciente apresentou hematoma biparietal e em outro, os dois hematomas ocorreram na região dos gânglios da base e no lobo parietal, mas em épocas diferentes. O menor hematoma mediu $8 \mathrm{ml}$ e o maior $115 \mathrm{ml}$, sendo o volume médio $42,2 \mathrm{ml}$.

Tabela 1. Distribuição dos casos segundo idade e gênero.

\begin{tabular}{ccccc}
\hline Idade (anos) & Masc. & Fem. & Total & $\%$ \\
\hline $20-29$ & 1 & 1 & 2 & 2,8 \\
$30-39$ & 2 & 2 & 4 & 5,7 \\
$40-49$ & 7 & 1 & 8 & 11,4 \\
$50-59$ & 13 & 6 & 19 & 27,1 \\
$60-69$ & 13 & 8 & 21 & 30,0 \\
$70-79$ & 9 & 5 & 14 & 20,0 \\
$80-89$ & 2 & - & 2 & 2,8 \\
Total & 47 & 23 & 70 & 100 \\
\hline
\end{tabular}

Os sintomas e sinais observados nos pacientes se acham nas Tabelas 4 e 5 . Entre os $62(86,1 \%)$ pacientes que apresentaram distúrbios da consciência, o estado de coma foi o achado mais frequente. Em $36,1 \%$

Tabela 2. Fatores de risco em 20 casos de óbito.

\begin{tabular}{lcc}
\hline Fatores de risco & $\mathrm{N}^{\circ}$ casos & $\%$ \\
Hematomas profundos & 17 & 85 \\
Coma & 15 & 75 \\
Distúrbios respiratórios & 11 & 55 \\
Idade (5 $5^{\mathrm{a}}$ e $6^{\mathrm{a}}$ décadas) & 11 & 55 \\
\hline
\end{tabular}


Tabela 3. Localização dos hematomas.

\begin{tabular}{lcc}
\hline Localização & $\mathrm{N}^{\circ}$ casos & $\%$ \\
\hline Gânglios da base & 17 & 31,4 \\
Lobar & 13 & 24,0 \\
Multilobar & 14 & 25,9 \\
Cerebelo & 10 & 18,5 \\
Invasão ventricular & 6 & 11,1 \\
\hline
\end{tabular}

destes, foram observados sinais de hipertensão intracraniana com herniação cerebral. Em relação à sintomatologia, o déficit motor contralateral, observado em $59(81,9 \%)$ pacientes, e o sinal de Babinski, obtido em 35 $(48,6 \%)$ outros, foram os achados mais frequentes.
Tabela 4. Sintomatologia por compressão/HIC.

\begin{tabular}{lcc}
\hline Achados & $\mathrm{N}^{\circ}$ Casos & $\%$ \\
\hline Cefaléia & 42 & 58,3 \\
Vômito & 22 & 30,5 \\
Edema de papila & 1 & 1,3 \\
Sonolência & 27 & 37,5 \\
Estupor & 6 & 8,3 \\
Coma & 29 & 40,2 \\
Midríase paralítica & 7 & 9,7 \\
Decorticação & 2 & 2,7 \\
Descerebração & 3 & 4,1 \\
Distúrbios respiratórios & 14 & 19,4 \\
\hline
\end{tabular}

Os valores médios de alguns fatores de risco cirúrgico como redução do nível de consciência, localização e volume do hematoma e sinais de herniação foram registrados em $79 \%$ dos pacientes operados nas duas primeiras décadas e em 40\% dos operados no período 1990-1999. Dos 59 pacientes que apresentavam déficit motor, em 24 (40,6\%), houve regressão parcial e apenas em 17 (28,8\%) a recuperação foi total.

A taxa de mortalidade (Tabela 6) registrou um total de 20 (27,7\%) óbitos, porém com significativa diferença percentual entre as três décadas do período em que ocorreu o tratamento.

\section{DISCUSSÃO}

A HICE origina-se da ruptura de vasos de pequeno calibre como as artérias estriadas, as artérias subcorticais lobares, os ramos paramedianos da artéria basilar e as artérias cerebelosas. Destas, as mais comprometidas são as artérias cerebelosas anterosuperior e anteroinferior. Em $80 \%$ dos casos, a coleção sanguínea localiza-se nos hemisférios cerebrais, ao passo que a distribuição dos $20 \%$ restantes ocorre no tronco cerebral e cerebelo, em percentual aproximado para ambas as

Tabela 5. Sintomatologia focal.

\begin{tabular}{lcc}
\hline Achados & $\mathrm{N}^{\circ}$ Casos & $\%$ \\
\hline Hemiparesia contralateral & 17 & 23,6 \\
Hemiplegia contralateral & 42 & 58,3 \\
Sinal de Babinski & 35 & 48,6 \\
Afasias & 4 & 5,5 \\
Hemianopsia homônima & 3 & 4,1 \\
Paralisia do olhar conjugado & 2 & 2,7 \\
Convulsão & 1 & 1,3 \\
Ataxia cerebelar & 7 & 9,7 \\
Sinais meningorradiculares & 2 & 2,7 \\
\hline
\end{tabular}
estruturas. Nos pacientes hipertensos, a hemorragia predomina na região dos gânglios da base ${ }^{3,6,8,11,13}$. Boudouresque et al., citados por Ferri-de-Barros e Bacheschi ${ }^{14}$, referem que as hemorragias ventriculares primárias são excepcionais. Comumente, segundo dados da literatura ${ }^{4,11,13,15,16}$, decorrem de invasão ventricular por sangramento profundo e

Tabela 6. Taxa de mortalidade dos 72 casos operados.

\begin{tabular}{lccc}
\hline Período & $\mathrm{N}^{\circ}$ Casos & Óbitos & $\%$ \\
\hline $70-79$ & 8 & 5 & 62,5 \\
$80-89$ & 11 & 4 & 36,3 \\
$90-99$ & 53 & 11 & 20,7 \\
Total & 72 & 20 & 27,7 \\
\hline
\end{tabular}


representam de $2,9 \%$ a 23,8\% dos casos. Hemorragias múltiplas e/ou recidivantes podem acontecer e, como relatam Neau et al. ${ }^{5}$, a localização lobar é predominante. A hemorragia é considerada pequena quando o volume é igual ou inferior a $20 \mathrm{ml}$; média, entre 20-40; e grande, quando superior a 40 $\mathrm{ml}^{13}$.

A hipertensão arterial sistêmica crônica está presente em cerca de $50 \%$ dos casos de $\mathrm{HICE}^{3,5,13}$. Este percentual aumenta nos casos de localização ganglionar, em que alcança cifras superiores a $70 \%{ }^{6,9}$. Em pacientes normotensos, a ocorrência de aumentos transitórios da pressão arterial devem ser pesquisados. Caplan, citado por Fisher ${ }^{1}$, relata que aumentos súbitos da pressão sanguínea dependem de várias causas como frio intenso, uso de drogas simpaticomiméticas e estimulação do nervo trigêmeo. Fessler et al. ${ }^{17}$ registraram complicações hemorrágicas em pacientes que fizeram uso crônico de cocaína e encontraram maior número de sangramentos nos portadores de malformações vasculares cerebrais. Na vigência de hemorragias recidivantes em pacientes idosos e, sobretudo, naqueles que apresentam quadro demencial, a possibilidade de angiopatia amilóide deve ser considerada $^{5}$. Em cerca de um terço dos casos, a HICE não tem causa conhecida ${ }^{4,13}$. A ocorrência de HICE depende, na maioria das vezes, das condições prévias da estrutura vascular. Ferri-de-Barros e Bacheschi ${ }^{14}$, citando Ojemann e Mohr, referem que as artérias cerebrais podem suportar pressões de até $1520 \mathrm{mmHg}$. Assim sendo, parece improvável a ruptura de vasos que mantêm suas paredes íntegras. Por outro lado, pacientes hipertensos crônicos comumente desenvolvem processo degenerativo das pequenas artérias, que resulta em lipo-hialinose, necrose fibrinóide e dilatações miliares conhecidas como aneurismas de Charcot-Bouchard ${ }^{18,19}$. Assim, o enfraquecimento da parede arterial cria condições para a sua ruptura, especialmente, quando associado ao aumento da pressão intravascular. Se a hemorragia acontece, a presença de sangue no parênquima cerebral produz alterações histopatológicas e bioquímicas que, segundo Kingman et al. e Kobarin et al., citados por $S^{S a m s o n}{ }^{2}$, são lesivas às células nervosas e provocam acentuado prejuízo de suas funções. O hematoma comprime, destrói e disseca o tecido cerebral. Substâncias de alta toxicidade como hemoglobina e partículas de ferro são liberadas. Na região perilesional, ocorrem alterações do fluxo sanguíneo cerebral e desenvolve-se edema vasogênico. Wallenfang et al. ${ }^{20}$ observaram que as imagens de hipodensidade que circundam o hematoma, mostradas pela TCC, apresentam expansões diferentes quando comparadas entre pacientes hipertensos e normotensos. Relacionando estes dados com os achados de trabalho experimental, realizado através de microscopia imunofluorescente, verificaram que, nos hipertensos entre o sétimo e o oitavo dia após a lesão hemorrágica, a menor extensão da área hipodensa está associada à maior dificuldade de drenagem local. Estes achados, na opinião dos autores, podem explicar a piora clínica apresentada por alguns pacientes hipertensos ao fim da primeira semana de doença.

A HICE é afecção grave, de instalação e evolução variáveis, dependendo do volume e da localização do hematoma. A sintomatologia predominante está representada pela síndrome de hipertensão intracraniana e o aparecimento de sinais focais ${ }^{3,4,6,8,13,14}$. Mohr et al..$^{9}$ observaram, em um grupo de 70 pacientes, início gradual em $63 \%$ e súbito em $34 \%$. Lin et al ${ }^{21}$ descrevem três casos de hemorragia cerebral de evolução lenta e progressiva, sugestiva de lesão tumoral, mesmo após a avaliação tomográfica. Comumente, os hematomas volumosos e profundamente situados ou aqueles que comprimem diretamente o tronco cerebral são responsáveis pelo desenvolvimento do estado de coma e da hipertensão intracraniana. Sinais sugestivos de herniação cerebral são constituídos pela instalação de midríase paralítica, decorticação, descerebração e alteração do ritmo respiratório. A sintomatologia focal reflete a disfunção de determinada área cerebral. Frequentemente, as hemorragias localizadas nos hemisférios cerebrais acompanham-se de prejuízo das funções motoras e/ou sensitivas como hemiplegia e hemianestesia contralaterais, hemianopsia homônima e paralisia do olhar conjugado. Afasia motora ou sensorial é achado frequente quando o hemisfério dominante é afetado. Por vezes, alguns sinais sugerem localizações específicas como maior comprometimento motor e desvio conjugado do olhar para o lado da lesão nas hemorragias putaminais, ou maior disfunção sensitiva e presença da síndrome de Parinaud nas hemorragias talâmicas. A hemianopsia homônima de aparecimento isolado, assim como o desenvolvimento de crises convulsivas favorecem o 
diagnóstico de infarto lobar. As hemorragias pontinas são caracterizadas por coma precoce, tetraplegia, pupilas puntiformes fotorreagentes e oscilações oculares (ocular bobbing). Alguns casos podem apresentar forma assimétrica com desvio conjugado dos olhos para a hemiplegia associado à paralisia dos nervos abducente e/ou facial. Em relação às hemorragias cerebelares, o que chama a atenção é a presença de incoordenação motora seguida de redução progressiva do nível de consciência, resultante do efeito compressivo sobre o tronco cerebral. A invasão de sangue para as cavidades ventriculares é caracterizada por cefaléia e sinais de irritação meningorradicular. Nestes casos, a ocorrência de sonolência e sinais de hipertensão intracraniana sugerem o desenvolvimento de hidrocefalia.

Por várias décadas, a angiografia cerebral foi o exame mais utilizado na avaliação das doenças cerebrovasculares. Atualmente, a sua principal indicação reside na identificação de malformações vasculares, particularmente, quando há suspeita de ruptura de aneurisma. A TCC tornou-se o exame de primeira escolha na exploração das doenças cerebrais por oferecer maiores possibilidades na identificação de vários tipos de lesões intracranianas e por apresentar maior sensibilidade e precocidade diagnóstica em relação à presença de sangue no tecido cerebral. Kinkel e Jacobs ${ }^{22}$ salientaram a importância da TCC ao mudar o diagnóstico de trombose cerebral para hemorragia em $43 \%$ dos casos.

Embora o tratamento da HICE seja motivo de muita discussão, algumas condutas devem ser tomadas independentemente da terapêutica escolhida. A hipertensão intracraniana deve ser reduzida através de medidas que diminuam o volume sanguíneo intracraniano como a manuntenção da cabeça do paciente em posição elevada e a hiperventilação, procurando-se manter a concentração de $\mathrm{pCO}_{2}$ entre 25-30 mmHg. A hipertensão arterial deve ser controlada com o emprego de drogas que evitem o aumento da volemia. A redução da pressão sanguínea deve ser moderada para não interferir com o fluxo sanguíneo cerebral, especialmente, durante a fase aguda da doença, quando podem ocorrer aumentos pressóricos transitórios em resposta ao reflexo de Cushing. É na definição do tratamento, se conservador ou cirúrgico, que reside a maior discordância. Lynch et al. ${ }^{6}$ e Waga et al. ${ }^{7}$ não observaram diferenças significativas nos índices de mortalidade em pacientes submetidos a tratamento conservador ou cirúrgico. Dourado et al. ${ }^{4}$, revisando 58 casos operados de hemorragia lobar, verificaram taxa de mortalidade de $10,3 \%$, mas apenas $20,2 \%$ deles apresentavam distúrbios de consciência. Broderick et al. ${ }^{23}$ analisaram 188 casos de hemorragia intracerebral e relatam que $25 \%$ dos 26 pacientes operados não sobreviveram, porém, no grupo que permaneceu em tratamento conservador, o obituário alcançou 46\%. Na avaliação das HICEs, alguns fatores são considerados de risco e devem, portanto, ser ponderados na escolha do tratamento. Entre os de maior importância, são citados idade avançada, hipertensão arterial, redução do nível de consciência, hematoma grande e de localização profunda, sinais de disfunção do tronco cerebral e péssimas condições físicas ${ }^{5,7,8,15}$. Nas hemorragias cerebelares, o nível de consciência é um dado valioso e, segundo Ojemann e Mohr, citados por Ferri-de-Barros e Bacheschi ${ }^{24}$, a cirurgia está indicada devido à imprevisibilidade da evolução clínica. Schaller et al. ${ }^{25}$ defendem o emprego de cirurgia estereotáxica e aplicação do ativador de plasminogênio tissular recombinante desde que sejam seguidos alguns critérios: idade inferior a 70 anos, redução do nível de consciência, hematoma com diâmetro mínimo de $3 \mathrm{~cm}$, tempo máximo decorrido de 72 horas e ausência de malformações vasculares e doenças hematológicas.

O grupo do presente estudo apresentava características clínicas semelhantes àquelas comumente observadas nas HICEs. Em $77 \%$ dos pacientes, a faixa etária prevalente situava-se entre os 50 e 70 anos, havendo ampla preponderância do gênero masculino e do grupo racial caucasóide. Apenas dois pacientes eram de cor preta, indício da baixa incidência do grupo negróide em nossa região. A hipertensão arterial ocorreu em número inferior àqueles relatados por alguns autores ${ }^{3,6,9,15}$ É possível que o número de hipertensos, registrado no presente estudo, esteja relacionado ao menor número de hemorragias localizadas na região dos gânglios da base. Em relação à sintomatologia, os

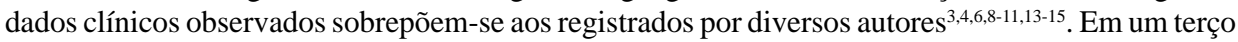
dos casos com hipertensão intracraniana, foram observados sinais de herniação cerebral, cuja presença teve grande importância na decisão do tratamento. Na análise dos resultados cirúrgicos, constatouse que os pacientes, operados no período 1990-1999, obtiveram significativa redução na taxa de 
mortalidade em comparação aos índices registrados nas duas décadas anteriores. Outrossim, observamos que os valores médios de alguns fatores, considerados de risco cirúrgico, ocorreram em número bem menor nos casos operados no último período. Além disso, é importante salientar que os 52 pacientes avaliados através da TCC foram aqueles pertencentes ao grupo de menor mortalidade. A inter-relação de todos estes dados mostra que a análise mais detalhada das características dos hematomas, através da neuroimagem, trouxe importante contribuição para a indicação adequada do procedimento cirúrgico. Neste grupo de pacientes, portanto, foram introduzidas modificações de técnica cirúrgica como craniectomias de pequenas dimensões $(2 \times 2 \mathrm{~cm}$ ou $3 \times 3 \mathrm{~cm})$ e o emprego de microscópio, fatores que, aliados aos supracitados, diminuíram a agressão ao parênquima cerebral, o tempo cirúrgico e a mortalidade.

Em nossa opinião, o tratamento cirúrgico está indicado nas hemorragias lobares que apresentam sinais de hipertensão intracraniana, nos casos complicados por hidrocefalia e nas hemorragias cerebelares com comprometimento do nível de consciência. Por outro lado, deve-se contra-indicar a aspiração do hematoma em face de coma profundo e naqueles com sinais de herniação cerebral e evidência de cisterna quadrigeminal fechada.

\section{REFERÊNCIAS}

1. Fisher M. Primary intracerebral and subarachnoid hemorrhage: an approach to diagnosis and therapy. Arq Neuropsiquiatr 1991;49:233-242.

2. Samson M. Hématome intracérébral spontané. Paris: Encycl Méd Chir 1991;17496 A ${ }^{10}, 1-6$.

3. Werneck LC, Scola RH, Ferraz LE. Hematomas intracerebrais espontâneos. Arq Neuropsiquiatr 1991;49:18-26.

4. Dourado MET, Freitas ML, Marti-Vilalta JL. Hemorragia cerebral lobar: clínica, etiologia, evolução. Revisão de 58 casos. Arq Neuropsiquiatr 1993;51:183-189.

5. Neau J-P, Ingrand P, Couderq C, et al. Recurrent intracerebral hemorrhage. Neurology 1997;49:106-113.

6. Lynch JC, Alves R, Ribeiro R, Lima JG. Hematomas intracerebrais hipertensivos. A propósito de 50 casos. Arq Neuropsiquiatr 1985;43:167-175.

7. Waga S, Miazaki M, Okada M, Tchio H, Matsushima S, Tanaka Y. Hypertensive hemorrhage: analysis of 182 pacients. Surg Neurol 1986;26:159-166.

8. Kobayashi S, Sato A, Kageyama Y, Nakamura H, Watanabe Y, Yamura A. Treatment of hypertensive cerebellar hemorrhage: surgical or conservative management ? Neurosurgery 1994;34:246-251.

9. Mohr JP, Caplan LR, Melski JW, et al. The Harvard cooperative stroke registry: a prospestive registry. Neurology 1978;28:754-762.

10. Ferreira NP, Chaves DL, Kraemer JL. Hematomas intraparenquimatosos espontâneos supratentoriais: a propósito de 28 casos. Arq Neuropsiquiatr 1980;38:367-374.

11. Kraemer JL, Bastos CAG, Brasil AVB, Paglioli E Neto, Ferreira NP. Hematomas intraparenquimatosos espontâneos: experiência com 134 casos. Arq Neuropsiquiatr 1992;50:10-15.

12. Bruno A, Carter S, Qualls C, Notte KB. Incidence of spontaneous intracerebral hemorrhage among hispanics and nonhispanic whites in New Mexico. Neurology 1996;47:405-407.

13. Kase CS, Williams JP, Wyatt DA, Mohr JP. Lobar intracerebral hematomas: clinical and CT analysis of 22 cases. Neurology 1982;32:1146-1150.

14. Ferri-de-Barros JE, Bacheschi LA. Hemorragia intraparenquimatosa espontânea: I. Conceito, incidência, etiologia e classificação. Arq Bras Neurocirurg 1983;2:291-299.

15. Walshe TM, Davis KR, Fisher CM. Thalamic hemorrhage: a computed tomography-clinical correlation. Neurology 1977;27:217-222.

16. Ferrera NP, Kraemer JL. Hematomas intracerebrais espontâneos: aspectos prognósticos em função da topografia. Arq Bras Neurocirurg 1991;10:123-130.

17. Fessler RD, Esshaki CM, Stankewitz RC, Johson RR, Diaz FG. The neurovascular complications of cocaine. Surg Neurol 1997;47:6-8.

18. Fisher CM. Lacuna strokes and infarcts: a review. Neurology 1982;32:871-876.

19. Utterback RA. Hemorrhagic cerebrovascular disease. In Baker AB (ed) Clinical neurology: Philadelphia: Harper \& Row, 1985;16:1-31.

20. Wallenfang T, Fries G, Ulrich P, Jantzen J-P. Tissue elastance and fluid conduction in normotensive and hypertensive intracerebral mass haematomas. Acta Neurochir (Wien) 1988;95:28-33.

21. Lin S-Z, Shih C-J, Wong Y-C, Tsoi S-H. Intracerebral hematoma simulating a new growth. Surg Neurol 1984;21:459-464.

22. Kinkel WR, Jacobs L. Computerized axial trasverse tomography in cerebrovascular disease. Neurology 1976;26:924-930.

23. Broderick J, Brott T, Tomsick T, Tew J, Duldmer J, Huster B. Management of intracerebral hemorrhage in a large metropolitan population. Neurosurgery 1994;34:882-887.

24. Ferri-de-Barros JE, Bacheschi LA. Hemorragia intraparenquimatosa espontânea II. Tratamento e prognóstico. Arq Bras Neurocirurg 1984;3:57-64.

25. Schaller C, Rohde V, Meyer B, Hossler W. Stereotatic puncture and lysis of spontaneous intracerebral hemorrhage using recombinant tissue - plasminogen activator. Neurosurgery 1995;36:328-335. 